\title{
Effect of Cilnidipine on Left Ventricular Diastolic Function in Hypertensive Patients as Assessed by Pulsed Doppler Echocardiography and Pulsed Tissue Doppler Imaging
}

\author{
Yukiko Onose, MD; Takashi Oki, MD; Hirotsugu Yamada, MD; \\ Kazuyo Manabe, MD; Yoshimi Kageji, MD; Masako Matsuoka, MD; \\ Takashi Yamamoto, MD; Tomotsugu Tabata, MD; \\ Tetsuzo Wakatsuki, MD; Susumu Ito, MD
}

\begin{abstract}
The purpose of the present study was to examine the mechanisms of improvement in left ventricular (LV) diastolic function in hypertensive patients treated with cilnidipine, a new and unique calcium antagonist that has both L-type and N-type voltage-dependent calcium channel blocking actions, using pulsed Doppler echocardiography and pulsed tissue Doppler imaging. The study comprised 35 untreated patients with essential hypertension (19 men and 16 women; mean age $65 \pm 10$ years). The peak early diastolic and atrial systolic transmitral flow velocities (E and A, respectively) and their ratio (E/A), and the peak early diastolic and atrial systolic motion velocities (Ew and Aw, respectively) of the LV posterior wall and their ratio (Ew/Aw) were determined in all patients before and after 1, 3 and 6 months on cilnidipine $(10 \mathrm{mg} /$ day). One month: Systolic and diastolic blood pressures were significantly decreased. E and E/A were significantly increased, whereas there were no significant changes in Ew and Ew/Aw. Three months: Ew and Ew/Aw were significantly increased compared to those before and 1 month after cilnidipine. Six months: E and E/A were significantly increased compared with before and 3 months after cilnidipine, and Ew and Ew/Aw were significantly increased compared with before cilnidipine. Moreover, the LV mass index was significantly decreased compared to that before cilnidipine. In summary, changes in LV diastolic performance in patients with essential hypertension following cilnidipine treatment were biphasic with an initial increase in early diastolic transmitral flow velocity and a later increase in early diastolic LV wall motion velocity. The initial and later changes can be related to an acute change in afterload and a later improvement in LV relaxation. (Jpn Circ J 2001; 65: 305-309)
\end{abstract}

Key Words: Hypertension; Left ventricular diastolic function; Pulsed tissue Doppler imaging; Transmitral flow velocity

【 eft ventricular (LV) systolic ${ }^{1}$ and diastolic ${ }^{2,3}$ functions are frequently impaired in hypertensive patients with LV hypertrophy. Because LV hypertrophy may become an important risk factor increasing the morbidity or mortality in ischemic heart disease, 4,5 many clinical studies have been conducted to evaluate morphological and functional improvements of the $\mathrm{LV}$ after administering various antihypertensive drugs $9^{6-9}$ Conversely, a number of studies on LV diastolic function in hypertensive patients show that diastolic dysfunction occurs before LV hypertrophy or systolic dysfunction, 2,3,10 Parameters obtained from the transmitral flow velocity pattern correlated closely with parameters obtained by left ventriculography ${ }^{11}$ and radionuclide angiography!2 Therefore, the transmitral flow velocity pattern is widely used to evaluate LV diastolic function in clinical settings. However, because the transmitral flow velocity pattern is influenced strongly by loading condition, such as left atrial pressure or aortic pressure, there is a limitation when this index is used to evaluate LV diastolic

(Received September 21, 2000; revised manuscript received December 27, 2000; accepted January 10, 2001)

The Second Department of Internal Medicine, School of Medicine, The University of Tokushima, Tokushima, Japan

Mailing address: Takashi Oki, MD, Second Department of Internal Medicine, School of Medicine, The University of Tokushima, 2-50 Kuramoto-cho, Tokushima 770-8503, Japan function in patients with elevated LV end-diastolic pressure!3,14 Recently, a tissue Doppler imaging (TDI) method has been developed in which large Doppler signals obtained from the ventricular wall can be selectively displayed as a color or pulsed Doppler image by eliminating small Doppler signals produced by the blood flow. This new technique is expected to provide valuable information on LV wall motion abnormalities in patients with heart disease.$^{15-17}$ Cilnidipine is a novel and unique 1,4-dihydropyridine derivative calcium antagonist that has a hypotensive action of slow-onset and prolonged duration, and has potent inhibitory action on L- and N-type voltage-dependent calcium channels. Clinical usefulness of this calcium antagonist is expected as a result from its heart-protecting action in addition to its antihypertensive effect! ${ }^{8,19}$ The present study was designed to evaluate the effect of cilnidipine on LV diastolic dysfunction in hypertensive patients, and to confirm the presence or absence of its beneficial effect.

\section{Methods}

\section{Study Population}

The study comprised 35 Japanese outpatients (19 men and 16 women aged $65 \pm 10$ years $($ mean $\pm S D)$ ) with essential hypertension who had not been treated previously with antihypertensive drugs. All the patients had a systolic blood 
pressure of $\geq 160 \mathrm{mmHg}$ or a diastolic blood pressure of $\geq 95$ $\mathrm{mmHg}$, or both, on at least 3 consecutive readings taken at the outpatient clinic of the hospital. The LV end-diastolic dimension and percent LV fractional shortening measured from M-mode echocardiography was $\leq 5.0 \mathrm{~cm}$ and $\geq 30 \%$, respectively. Furthermore, all the patients displayed LV impaired diastolic filling; namely, the peak atrial systolic velocity (A) was greater than the peak early diastolic velocity $(\mathrm{E})(\mathrm{E} / \mathrm{A}<1)$ in the transmitral flow velocity pattern obtained by pulsed Doppler echocardiography. On the basis of their medical history, a physical examination, routine laboratory studies and screening echocardiography, none of the patients in the present study had secondary hypertension, myocardial infarction, angina pectoris, congenital heart disease, chronic renal failure, or significant valvular heart disease.

\section{Study Protocol}

The purpose of the study was explained and informed consent to participate was obtained from each patient. Cilnidipine was administered to all patients at a daily dose of $10 \mathrm{mg}$ for 6 consecutive months. None of the patients
A

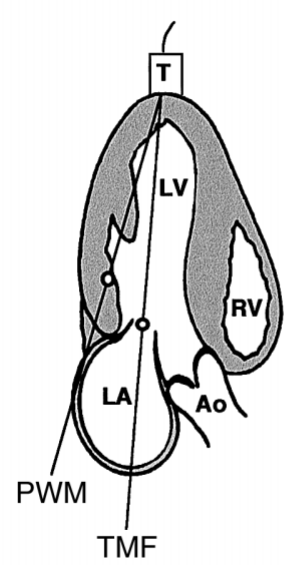

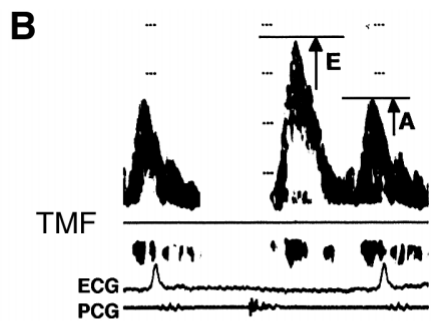

C

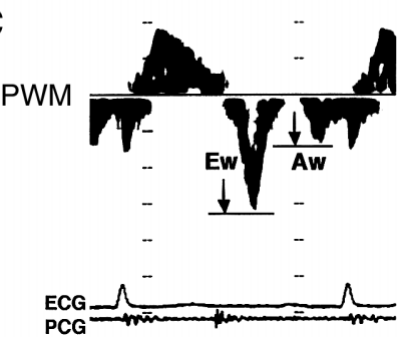

Fig 1. Pulsed Doppler echocardiographic recording of transmitral flow (TMF) velocity and pulsed tissue Doppler recording of left ventricular posterior wall motion (PWM) velocity in the apical longaxis view of the left ventricle (LV). Typical TMF (B) and PWM (C) velocity patterns are shown in the right panel. LA, left atrium; Ao, ascending aorta; RV, right ventricle; $\mathrm{T}$, transducer; $\mathrm{E}$, peak early diastolic TMF velocity; A, peak atrial systolic TMF velocity; Ew, peak early diastolic PWM velocity; Aw, peak atrial systolic PWM velocity; ECG, electrocardiogram; PCG, phonocardiogram.

received any medication other than cilnidipine to treat their hypertension. During this period, the patients were asked to come to the hospital every 2 weeks to assess compliance and for blood pressure and pulse/heart rate measurements.

Before and after 1, 3 and 6 months of treatment with cilnidipine, plasma noradrenaline levels were measured. To prevent an increase in noradrenaline levels resulting from pain, blood samples were collected after a 30 min rest in the supine position.

\section{M-Mode and Pulsed Doppler Echocardiography}

Before and after 1, 3 and 6 months of treatment with cilnidipine, M-mode and pulsed Doppler echocardiograms were recorded on a stripchart recorder at a speed of $50 \mathrm{~mm} / \mathrm{s}$ with simultaneous electrocardiogram and phonocardiogram tracings for all patients.

On the M-mode LV echocardiogram, the LV end-diastolic dimension (LVDd), LV end-systolic dimension (LVDs) and end-diastolic thicknesses of the ventricular septum (VSth) and the posterior wall (PWth) were measured. The percent fractional shortening (\%FS) of LV was calculated using LV internal dimensions (LVDd and LVDs). Left ventricular mass (LVM) was determined using the following equation: ${ }^{20}$

$$
\begin{array}{r}
\mathrm{LVM}=1.04 \times\left[\left(\mathrm{LVDd}+\mathrm{PWth}+\mathrm{VSth}^{3}-\right.\right. \\
\left.\mathrm{LVDd}^{3}\right]-13.6
\end{array}
$$

The LVM index (LVMI) was determined by dividing each measurement by the body surface area.

A sample volume was set at the mitral valve orifice in the long-axis view of the LV recorded from the cardiac apex, and the transmitral flow velocity patterns were recorded using a commercially available Toshiba SSA-380A (Toshiba Corporation, Tokyo, Japan; $3.75 \mathrm{MHz}$ probe) (Fig 1A). From the obtained pattern, the peak early diastolic velocity (E) and peak atrial systolic velocity (A), and their ratio (E/A) were determined (Fig 1B).

\section{Pulsed TDI}

In the apical long-axis view of the LV, sample volumes were set at the endocardial portion of the middle site of the LV posterior wall (Fig 1A). The motion velocity patterns were recorded by the pulsed Doppler method using a Toshiba SSA-380A instrument. From the obtained patterns, the peak early diastolic wall motion velocity $(\mathrm{Ew})$ and peak atrial systolic wall motion velocity $(\mathrm{Aw})$, and their ratio (Ew/Aw) were determined (Fig 1C).

The interobserver variability in measurements of pulsed Doppler and pulsed TDI indices was calculated as the difference in 2 measurements performed in the same patient by 2 different observers divided by the mean value. The interobserver variability was $3-8 \%$ for E, A, E/A, Ew, $\mathrm{Aw}$, and Ew/Aw. The intraobserver variability was calcu-

Table 1 Clinical Findings

\begin{tabular}{lcccc}
\hline \hline & Baseline & 1 month & 3 months & 6 months \\
\hline Age (years) & $65 \pm 10$ & - & - & - \\
M/F & $19 / 16$ & - & - & - \\
Body height $(\mathrm{cm})$ & $158 \pm 3$ & - & - & - \\
Body weight $(\mathrm{kg})$ & $59 \pm 4$ & - & - & - \\
Heart rate $($ beats/min) & $59 \pm 7$ & $60 \pm 11$ & $59 \pm 9$ & $60 \pm 9$ \\
Systolic blood pressure $(\mathrm{mmHg})$ & $174 \pm 17$ & $148 \pm 10^{* * *}$ & $143 \pm 9^{* * *}$ & $142 \pm 11^{* * *}$ \\
Diastolic blood pressure $(\mathrm{mmHg})$ & $96 \pm 10$ & $82 \pm 16^{*}$ & $80 \pm 6^{*}$ & $78 \pm 8^{*}$ \\
Noradrenaline $(\mathrm{ng} / \mathrm{ml})$ & $0.5 \pm 0.3$ & $0.5 \pm 0.2$ & $0.4 \pm 0.1$ & $0.4 \pm 0.2$ \\
\hline
\end{tabular}

$*_{p}<0.05, * * * p<0.0001$ vs Baseline. 
Table 2 M-Mode and Pulsed Doppler Echocardiographic Variables

\begin{tabular}{lcccc}
\hline \hline & Baseline & 1 month & 3 months & 6 months \\
\hline $\operatorname{LVDd}(\mathrm{cm})$ & $4.7 \pm 0.3$ & $4.7 \pm 0.3$ & $4.6 \pm 0.3$ & $4.7 \pm 0.4$ \\
$\operatorname{LVDs}(\mathrm{cm})$ & $2.8 \pm 0.4$ & $2.7 \pm 0.4$ & $2.8 \pm 0.2$ & $2.7 \pm 0.3$ \\
$\% F S(\%)$ & $39 \pm 3$ & $40 \pm 4$ & $39 \pm 5$ & $41 \pm 3$ \\
$\operatorname{LVMI}\left(\mathrm{g} / \mathrm{m}^{2}\right)$ & $131 \pm 22$ & $132 \pm 17$ & $129 \pm 18$ & $126 \pm 20^{*}$ \\
$E(\mathrm{~cm} / \mathrm{s})$ & $55 \pm 8$ & $65 \pm 10^{*}$ & $63 \pm 8^{*}$ & $69 \pm 7^{*+}$ \\
$A(\mathrm{~cm} / \mathrm{s})$ & $73 \pm 7$ & $74 \pm 11$ & $72 \pm 8$ & $71 \pm 8$ \\
$E / A$ & $0.7 \pm 0.1$ & $0.9 \pm 0.2^{* *}$ & $0.9 \pm 0.1 * *$ & $1.0 \pm 0.1^{* * *+}$ \\
\hline
\end{tabular}

${ }^{*} p<0.05, *^{*} p<0.01,{ }^{* *} p<0.0001$ vs Baseline, ${ }^{+} p<0.05$ vs 3 months. LVDd, left ventricular end-diastolic dimension; LVDs, left ventricular end-systolic dimension; \%FS, percent fractional shortening of LV; LVMI, left ventricular mass index; $E$, early diastolic transmitral flow velocity; A, atrial systolic transmitral flow velocity; $E / A$, the ratio of $E$ to $A$.

Table 3 Pulsed Tissue Doppler Imaging Variables

\begin{tabular}{lcccc}
\hline \hline & Baseline & 1 month & 3 months & 6 months \\
\hline$E w(\mathrm{~cm} / \mathrm{s})$ & $8.6 \pm 1.8$ & $8.8 \pm 1.9$ & $11.3 \pm 2.1^{* * *+++}$ & $11.8 \pm 2.1^{* * *++}$ \\
$A w(\mathrm{~cm} / \mathrm{s})$ & $9.3 \pm 1.3$ & $9.5 \pm 1.5$ & $9.7 \pm 1.6$ & $9.5 \pm 1.6$ \\
$E w / A w$ & $0.9 \pm 0.2$ & $0.9 \pm 0.4$ & $1.2 \pm 0.3^{* *++}$ & $1.3 \pm 0.4^{* *++}$ \\
\hline
\end{tabular}

${ }^{* *} p<0.001,{ }^{* * *} p<0.0001$ vs Baseline, ${ }^{++} p<0.001,{ }^{++} p<0.0001$ vs 1 month. Ew, early diastolic left ventricular wall motion velocity; $A w$, atrial systolic left ventricular wall motion velocity; Ew/Aw, the ratio of Ew to Aw.
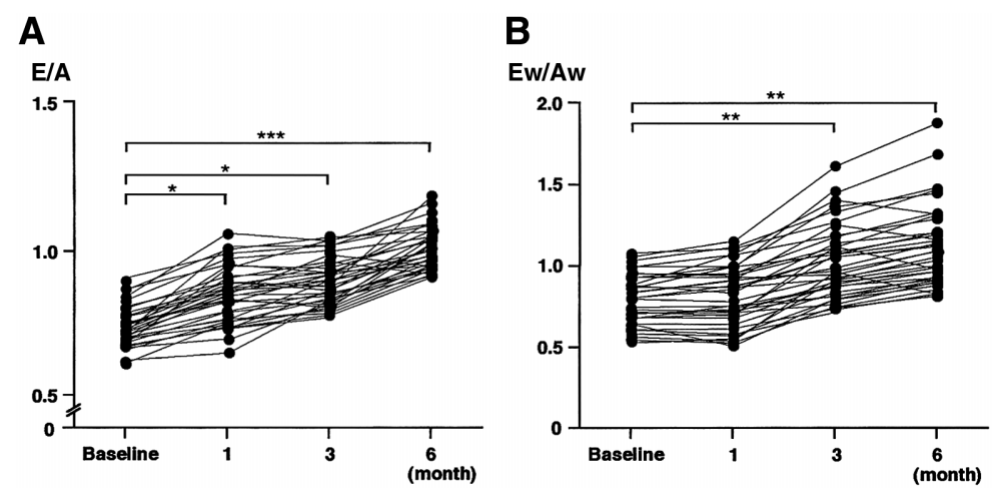

Fig 2. Temporal changes in the ratios of peak early diastolic to atrial systolic velocity of the transmitral flow and left ventricular posterior wall motion velocity patterns (E/A and Ew/Aw, respectively) during the treatment with cilnidipine in patients with hypertension. $* \mathrm{p}<0.01, * * \mathrm{p}<0.001, * * * \mathrm{p}<0.0001$ vs baseline. lated as the difference between 2 measurements performed in the same patient by 1 observer divided by the mean value. The intraobserver variability was $2-6 \%$ for the above indices.

\section{Statistical Analysis}

The mean of 5 consecutive heart beats was used for each measurement. Values are expressed as the mean \pm SD. Mean values for the clinical, M-mode and pulsed Doppler echocardiographic and pulsed TDI variables before and after administration of cilnidipine were compared with a 2-factor analysis of variance (ANOVA) for repeated measurements. A $p$ value $<0.05$ was considered statistically significant.

\section{Results}

\section{Clinical Characteristics}

Before administering cilnidipine, the mean systolic and diastolic blood pressures were $174 \pm 17 \mathrm{mmHg}$ and $96 \pm 10$ $\mathrm{mmHg}$, respectively. Left ventricular hypertrophy (LVMI $\geq$ $135 \mathrm{~g} / \mathrm{m}^{2}$ ) was observed in 7 of the 35 patients, and the mean value for LVMI was $131 \pm 22 \mathrm{~g} / \mathrm{m}^{2}$. There were no side-effects during treatment with cilnidipine. Systolic and diastolic blood pressures were significantly lower after 1, 3 and 6 months of treatment compared with baseline values (Table 1). However, there were no significant differences in systolic and diastolic blood pressures among values obtained after 1, 3 and 6 months of treatment. Heart rate and plasma noradrenaline levels did not significantly change during treatment.

\section{M-Mode Echocardiographic Variables}

There were no significant changes in LVDd, LVD, and $\% \mathrm{FS}$ of the LV during treatment compared with baseline values (Table 2). Left ventricular mass index tended to be lower after 3 months of treatment, and it was significantly lower after 6 months.

\section{Pulsed Doppler Echocardiographic and Pulsed TDI Variables}

The peak early diastolic transmitral flow velocity (E) was significantly increased after 1, 3 and 6 months on cilnidipine compared to baseline values, and was significantly increased after 6 months compared to after 3 months of treatment (Table 2). There was no significant change in the peak atrial systolic transmitral flow velocity (A) during the course of treatment. The E/A ratio was significantly increased after 1, 3 and 6 months of cilnidipine treatment compared to baseline values, and was significantly increased after 6 months compared to after 3 months of treatment (Fig 2A).

The peak early diastolic motion velocity (Ew) and the 
ratio of Ew to peak atrial systolic motion velocity (Aw) of the LV posterior wall (Ew/Aw) were significantly increased after 3 and 6 months of cilnidipine treatment compared to baseline and values after 1 month of treatment (Table 3; Fig 2B). The Aw did not significantly change during the course of treatment with cilnidipine.

\section{Discussion}

In the present study, cilnidipine was administered to patients with essential hypertension for 6 months and the following results were obtained: (i) cilnidipine significantly decreased systolic and diastolic blood pressures without changing heart rate; (ii) although there were no significant changes in LV end-diastolic and end-systolic dimensions, LVMI was significantly decreased after 6 months of cilnidipine treatment; (iii) when \%FS was used as an index to evaluate LV systolic pump function, there were no significant changes in \%FS during treatment with cilnidipine; and (iv) indices on LV diastolic function obtained from transmitral flow and LV wall motion velocity patterns significantly improved during treatment with cilnidipine. When temporal changes in these indices were assessed, transmitral flow velocity variables were found to have improved first (after 1 month of cilnidipine treatment), and then LV wall motion velocity variables subsequently improved (after 3 months of cilnidipine treatment).

\section{Antihypertensive Agent}

Cilnidipine is a dual calcium channel antagonist that has potent inhibitory action on both L- and N-type voltagedependent calcium channels. Based on these characteristics, it is reported that cilnidipine exhibits a vasodilative action as well inhibits reflex stimulation of the sympathetic nervous system that occurs immediately after medication. In recent studies, it was demonstrated that cilnidipine suppresses cardiac sympathetic overactivity in patients with essential hypertension, while amlodipine has little suppressive effect 18 and that heart rate was significantly lower during treatment with cilnidipine than during treatment with nisoldipine in both morning and afternoon segments 19 These results suggest that cilnidipine is superior as an antihypertensive agent as it also has heart-protective effects.

\section{Left Ventricular Hypertrophy}

An experimental study has previously clarified that biochemical, morphological and functional changes in LV myocardium resulting from pressure overload are reversed after normalization of afterload ${ }^{21}$ As demonstrated previously ${ }^{6-8,22}$ LV hypertrophy also improved in the present study after antihypertensive treatment with cilnidipine. Regarding the time course of regression of LV hypertrophy, it was reported that regression was initially observed after 1 month of treatment and then, after 4 months of treatment, LV hypertrophy completely improved6 However, it is also reported that LV hypertrophy gradually improved over 3 years? Therefore, the results of the present study, which show LV hypertrophy was improved after 6 months of treatment, do not significantly differ from those of previous studies. However, the degree of regression of LV hypertrophy $(7 \%)$ was slightly lower than that reported in other studies (18-33\%), , $^{\circ}$ It is difficult to simply compare the degree of regression of LV hypertrophy because there are differences in the age of patients, duration of LV hypertrophy, and types of antihypertensive drugs used.

\section{Left Ventricular Diastolic Function}

It was previously clarified that the indices of LV diastolic function obtained by transmitral flow velocity pattern correlated satisfactorily with those obtained by left ventriculography ${ }^{11}$ or radionuclide angiography!2 Therefore, LV diastolic function is widely and routinely evaluated in clinical settings using this method. However, the transmitral flow velocity pattern is influenced by loading conditions, particularly preload, resulting in pseudonormalization $(\mathrm{E} / \mathrm{A}>1)$ of the transmitral flow velocity in patients with elevated LV end-diastolic pressure!4,17 Various factors, such as LV myocardial relaxation, pressure difference between the LV and left atrium, and LV compliance, are related to LV filling during early diastole ${ }^{23,24}$ Therefore, there is a limitation when LV diastolic function is evaluated by the transmitral flow velocity pattern alone! ${ }^{13,14,17}$

Conversely, it was confirmed that early diastolic indices from pulsed TDI were not relatively influenced by preload, and correlated negatively with time constant from the LV pressure decay at isovolumic diastole (tau) ${ }^{17}$ and LV filling pressure 25 It was clarified that early diastolic wall motion velocity obtained by pulsed TDI was decreased both along the short and long axes of the LV in hypertensive patients with LV diastolic dysfunction ${ }^{26}$ Moreover, using simultaneous recording of LV wall motion velocity by pulsed TDI and transmitral flow velocity, it has been clarified that elastic recoil of the myocardium promotes LV filling in normal subjects, and LV filling begins during active relaxation of the LV myocardium in patients with elevated LV end-diastolic pressure? ${ }^{7}$

In the present study, it was most interesting that the $\mathrm{E}$ and E/A obtained from the transmitral flow velocity pattern improved first during treatment with cilnidipine, and then the Ew and Ew/Aw obtained from pulsed TDI subsequently improved. These results suggest that LV early diastolic filling initially improved due to the decrease in blood pressure (afterload) induced by cilnidipine and, thereafter, active relaxation of the LV myocardium gradually improved. These results also demonstrate that cilnidipine has an important myocardial-protecting action in addition to its antihypertensive effect.

Besides a decrease in afterload, the improvement in coronary microcirculation during cilnidipine administration is considered to be the improvement mechanism of $\mathrm{LV}$ diastolic function. It was demonstrated previously that the coronary microcirculation of LV myocardium is impaired in hypertensive patients with hypertrophy, ${ }^{28}$ and that the increase in coronary blood flow that was observed during treatment with nifedipine is important as an improvement mechanism of LV diastolic function? Based on this evidence, improvement in coronary microcirculation during cilnidipine administration might contribute to improved LV diastolic function.

\section{Study Limitations}

Despite the advantages of evaluating diastolic LV function by pulsed TDI, analyses of the present study still have some limitations. First, we measured the motion velocity only at the endocardial portion of the LV wall and, therefore, could not calculate the transmyocardial velocity gradient ${ }^{16,17}$ Consequently, disease-associated influences on the motion of the whole heart cannot be ruled out ${ }^{17}$ However, motion velocity at the endocardial or mid-wall portion is generally higher than that at the epicardial portion. Furthermore, the goal of the present study was not to obtain absolute values 
of the various parameters in patients with different conditions, but to assess the differences in the parameters derived from pulsed TDI during the course of administration of cilnidipine. Therefore, in the present study, the determination of motion velocity only at the endocardial portions has no clinical impact 17 Moreover, because regional LV wall motion velocity was measured by the aforementioned method, evaluation of global LV function using only the variable of the LV posterior wall is not appropriate in patients with regional LV asynergy. However, none of the patients in the present study revealed regional LV asynergy or asymmetric septal hypertrophy.

\section{References}

1. Borer JS, Jason M, Devereux RB, FischerJ, Green MV, Bacharac SL, et al: Function of the hypertrophied left ventricle at rest and during exercise: Hypertension and aortic stenosis. Am J Med 1983; 75(Suppl $3 \mathrm{~A}): 34-39$

2. Inouye I, Massie B, Loge D, Topic N, Silverstein D, Simpson P, et al: Abnormal left ventricular filling: An early finding in mild to moderate systemic hypertension. Am J Cardiol 1984; 53: 120-126

3. Fouad FM, Slominski JM, Tarazi RC: Left ventricular diastolic function in hypertension: Relation to left ventricular mass and systolic function. J Am Coll Cardiol 1984; 3: 1500-1506

4. Koren MJ, Devereux RB, Casale PN, Savage DD, Laragh JH: Relation of left ventricular mass and geometry to morbidity and mortality in uncomplicated essential hypertension. Ann Intern Med 1991; 114: $345-352$

5. Levy D, Garrison RJ, Savage DD, Kannel WB, Castelli WP: Prognostic implications of echocardiographically determined left ventricular mass in the Framingham heart study. N Engl J Med 1990; 322: $1561-1566$

6. Habib GB, Mann DL, Zoghbi WA: Normalization of cardiac structure and function after regression of cardiac hypertrophy. Am Heart $J$ 1994; 128: $333-343$

7. Nakashima Y, Nii T, Ikeda M, Arakawa K: Role of left ventricular regional nonuniformity in hypertensive diastolic dysfunction. $J \mathrm{Am}$ Coll Cardiol 1993; 22: 790-795

8. Guller B, Hall J, Reeves RL: Cardiac effects of trandolapril in hypertension. Am Heart J 1993; 125: 1536-1541

9. Betocchi S, Cuocolo A, Pace L, Chiariello M, Trimarco B, Alfano B, et al: Effects of intravenous verapamil administration on left ventricular diastolic function in systemic hypertension. Am J Cardiol 1987; 59: $624-629$

10. Hartford M, Wikstrand J, Wallentin I, Ljungman S, Wilhelmsen L, Berglund G: Diastolic function of the heart in untreated primary hypertension. Hypertension 1984; 6: 329-338

11. Rockey R, Kuo LC, Zoghbi WA, Limacher MC, Quinones MA: Determination of parameters of left ventricular diastolic filling with pulsed Doppler echocardiography: Comparison with cineangiography. Circulation 1985; 71: 543-550

12. Spirito P, Maron BJ, Bonow RO: Noninvasive assessment of left ventricular diastolic function: Comparative analysis of Doppler echocardiographic and radionuclide angiographic technique. $J$ Am Coll Cardiol 1986; 7: 518-526

13. Ishida Y, Meisner JS, Tsujioka K, Gallo JI, Yoran C, Frater RWM, et al: Left ventricular filling dynamics: Influence of left ventricular relaxation and left atrial pressure. Circulation 1986; 74: 187-196

14. Thomas JD, Flachskampf FA, Chen C, Guererro JL, Picard MH, Levine RA, et al: Isovolumic relaxation time varies predictably with its time constant and aortic and left atrial pressure: Implications for the noninvasive evaluation of ventricular relaxation. Am Heart $J$ 1992; 124: 1305-1313

15. Isaaz K, Thompson A, Ethevenot G, Cloez JL, Brembilla B, Pemot $\mathrm{C}$ : Doppler echocardiographic measurement of low velocity motion of left ventricular posterior wall. Am J Cardiol 1989; 64: 66-75

16. Yamazaki N, Mine Y, Sano A, Hirama M, Miyatake K, Yamagishi $\mathrm{M}$, et al: Analysis of ventricular wall motion using color-coded tissue Doppler imaging system. Jpn J Appl Physiol 1994; 33: 3141-3146

17. Oki T, Tabata T, Yamada H, Wakatsuki T, Shinohara H, Nishikado A, et al: Clinical application of pulsed Doppler tissue imaging for assessing abnormal left ventricular relaxation. Am J Cardiol 1997; 79: 921-928

18. Sakata K, Shirotani M, Yoshida H, Nawada R, Obayashi K, Togi K, et al: Effects of amlodipine and cilnidipine on cardiac sympathetic nervous system and neurohormonal status in essential hypertension. Hypertension 1999; 33: 1447-1452

19. Minami J, Ishimitsu T, Higashi T, Numabe T, Matsuoka H: Comparison between cilnidipine and nisoldipine with respect to effects on blood pressure and heart rate in hypertensive patients. Hypertens Res 1998; 21: 215-219

20. Devereux RB, Reichek N: Echocardiographic determination of left ventricular mass in man. Circulation 1977; 55: 613-618

21. Marino TA, Brode E, Lauva IK, Kent RL, Cooper GI: Reversibility of the structural effects of pressure overload hypertrophy of cat right ventricular myocardium. Anat Rec 1986; 214: 141-147

22. Dahlof B, Pennert K, Hansson L: Reversal of left ventricular hypertrophy in hypertensive patients: A meta-analysis of 109 treatment studies. Am J Hypertens 1992; 5: 95-110

23. Grossman W, McLaurin LP: Diastolic properties of the left ventricle. Ann Intern Med 1976; 84: 316-326

24. Glantz SA, Parmley WW: Factors which affect the diastolic pressurevolume curve. Circ Res 1978; 42: 171-177

25. Nagueh SF, Middleton KJ, Kopelen HA, Zoghb WA, Quinones MA: Doppler tissue imaging: A noninvasive technique for evaluation of left ventricular relaxation and estimation of filling pressures. J Am Coll Cardiol 1997; 30: 1527-1533

26. Oki T, Tabata T, Yamada H, Wakatsuki T, Mishiro Y, Abe M, et al: Left ventricular diastolic properties of hypertensive patients measured by pulsed tissue Doppler imaging. J Am Soc Echocardiogr 1998; 11: $1106-1112$

27. Onose Y, Oki T, Tabata T, Yamada H, Ito S: Assessment of the temporal relationship between left ventricular relaxation and filling during early diastole using pulsed Doppler echocardiography and tissue Doppler imaging. Jpn Circ J 1999; 63: 209-215

28. Houghton JL, Frank MJ, Carr AA, Dohlen TW, Prisant LM: Relations among impaired coronary flow reserve, left ventricular hypertrophy and thallium perfusion defects in hypertensive patients without obstructive coronary artery disease. J Am Coll Cardiol 1990; 15: $43-51$ 